\title{
The mathematical model of producing winter diesel fuel (including logistic costs)
}

\author{
Valentin Nikonorov ${ }^{1, *}$, Andrei Kutuzov ${ }^{1}$, Viktor Nikonorov ${ }^{2}$, Irina Bagaeva ${ }^{1}$, and Anna \\ Letta $^{3}$ \\ ${ }^{1}$ Peter the Great St. Petersburg Polytechnic University, St. Petersburg, Russia \\ 2 JSC Lengiprostroy, St. Petersburg, Russia \\ ${ }^{3}$ University of Luxembourg, Faculty of Law, Economics and Finance, Luxembourg
}

\begin{abstract}
The authors reviewed the statistics of winter diesel fuel consumption in the Russian Federation for 2000-2017, revealed a positive growth rate. On this fact, the relevance of the study was substantiated. The complexity and importance of the refining process leads to the need for digitalization of oil refineries. The object of the study is obtaining winter diesel fuel, taking into account transport costs. The subject of the study is digitalization of the oil refining process on the base of constructing the mathematical model for obtaining winter diesel fuel, taking into account delivery costs. Main research methods: retrospective data analysis, synthesis, comparison, linear programming methods. The authors considered two stages of winter diesel fuel production: dewaxing a mixture of stages of hydrocarbon raw materials on zeolite and subsequent compounding. For each stage, the authors compiled a system of equations describing the process. The authors also compiled a target function for obtaining winter diesel fuel, taking into account the costs of receiving and delivering. The obtained mathematical model can be used to ensure the required quality of winter diesel fuel (in terms of sulfur content, density and flash point).
\end{abstract}

\section{Introduction}

Relevance. The main part of the territory of Russia is located between 70 and 50 degrees north latitude. $20 \%$ of the territory of the Russian Federation is located beyond the Arctic Circle. The need for grades of winter diesel fuel (hereinafter referred to as WDF) is becoming obvious. This is confirmed by statistical data (Table 1).

Table 1. Consumption of WDF for 2000-2017[1] (compiled by the authors).

\begin{tabular}{|c|c|c|}
\hline No. & Year & Diesel fuel, million tons \\
\hline 1 & 2000 & 9.4 \\
\hline 2 & 2001 & 9.0 \\
\hline 3 & 2002 & 8.7 \\
\hline 4 & 2003 & 8.8 \\
\hline 5 & 2004 & 9.1 \\
\hline
\end{tabular}

\footnotetext{
*Corresponding author: nikanorv@mail.ru
} 


\begin{tabular}{|c|c|c|}
\hline 6 & 2005 & 8.9 \\
\hline 7 & 2006 & 9.7 \\
\hline 8 & 2007 & 10.2 \\
\hline 9 & 2008 & 12.4 \\
\hline 10 & 2009 & 10.7 \\
\hline 11 & 2010 & 11.2 \\
\hline 12 & 2011 & 12.7 \\
\hline 13 & 2012 & 13.2 \\
\hline 15 & 2013 & 12.7 \\
\hline 16 & 2014 & 12.2 \\
\hline 17 & 2015 & 12.7 \\
\hline 18 & 2016 & 23.4 \\
\hline Growth rate, $\%$ & 2017 & 13.3 \\
\hline
\end{tabular}

Accordingly, the task of increasing the production of WDF acquires an actual value. Refining processes are quite complex. Digitalization of oil refining processes means, according to the authors, the construction and solution of the mathematical model for obtaining WDF (hereinafter referred to as MMWDF). That will identify possible resources for increasing the volume of WDF production and improving the quality of WDF.

Object of study is producing WDF. The subject of research is digitalizing oil-refining processes on the base of the mathematical model for obtaining WDF. The purpose of the study is to build this mathematical model.

\section{Materials and Methods}

In [2], it is proposed a mathematical model for the production of diesel fuel (hereinafter referred to as DF) based on the kinetics of the process (the rate of change in the molar concentration as a function of the reaction rate). A system of linear ordinary differential equations of the form:

$$
\frac{d C_{i}}{d t}=\sum_{j=1}^{n} \alpha_{j} W_{j}, i=1: m
$$

where:

$\mathrm{C}_{\mathrm{i}}$ is the molar concentration of the $\mathrm{i}$-th component;

$\mathrm{W}_{\mathrm{j}}$ is the rate of the $\mathrm{j}$-th reaction, $\mathrm{mol} /\left(\mathrm{m}^{3} \cdot \mathrm{s}\right)$;

$\alpha_{\mathrm{j}}=0$ or $\alpha_{\mathrm{j}}=1$

According to the authors, the quality indicators of diesel fuel are not taken into account.

In [3], Belinskaya N.S. investigates the processes of producing distillates with low boiling points based on atmospheric gasoils. In the opinion of the authors, this model does not take into account the chemical and technological features of obtaining diesel fuel.

In [4], a team of authors analyzes the main characteristics of winter diesel fuel depending on input components. Accordingly, the following are taken as basic components: denormalizate, diesel fraction with corresponding temperatures of the beginning and the end of boiling, hydrotreated winter diesel fuel. Each of the components contributes to the quality of winter diesel fuel obtained after compounding. Based on this idea, the researchers apply the problem of multi-criteria optimization. In our opinion, the obtained mathematical model can be run according to four well-known methods for solving multi-criteria optimization problems (criteria convolution, main criterion method, ideal point method, concessions method) and then choose the best method.

In [5], researchers propose using heavy fractions to obtain diesel fuel. In this case, on the one hand, there is a certain saving, since high-boiling oil fractions do not need additional purification when producing heavy diesel, but, on the other hand, the 
technological process imposes increased requirements on the quality of the distillation column. Here, in our opinion, the study can be supplemented again by using the method of multi-criteria optimization. Moreover, we must remember that heavy oil fractions contain an increased amount of sulfur (directly sulfur, mercaptans, sulfides, disulfides), which must be removed as much as possible (desulfurization).

In [6] and [7], mathematical models of catalytic dewaxing are proposed. In the process of catalytic dewaxing, catalytic cracking and isomerization of normal alkanes are also observed. Isomerization and cracking leads to the emergence of new hydrocarbon molecules with a lower molar mass, which also contributes to the production of winter diesel fuel (which does not solidify at low temperatures).

In [8], the researchers considered a mathematical model for intensifying the mixing of components to obtain motor fuel.

In [9], a team of researchers considers a rather important indicator of the quality of winter diesel fuel, the cetane number. The cetane number depends on the content of hexadecane $\left(\mathrm{C}_{16} \mathrm{H}_{34}\right)$ in winter diesel fuel and characterizes the flammability of winter diesel fuel (the relationship between the flammability of winter diesel fuel and the cetane number is direct). The researchers propose an original method for predicting the cetane number depending on the starting components of winter diesel fuel.

In [10], researchers ask the question of reducing the sulfur content in diesel fractions since sulfur derivatives are highly corrosive, which reduces engine life. To solve the problem of desulfurization, quantum-chemical calculations are proposed.

In [11], researchers indicate the way of desulfurization of winter diesel fuel at the expense of hydrogen-containing gas. Varying the hydrogen gas indicators can increase the desulfurization effect.

In [12], researchers analyze the process of catalytic dewaxing. Catalytic dewaxing speeds up the process and improves the quality of the components obtained, but the cost of the catalyst should be taken into account.

In [13], researchers consider the issue of intensification of dewaxing due to the redistribution of hydrogen. In this case, according to the authors, the methods of optimal control can be applied.

In [14], researchers analyze the process of catalytic dewaxing, for which they used a mathematical model of the catalyst operation in different technological modes.

In [15], researchers apply mathematical modeling to maximize the yield of denormalizate after dewaxing.

In [16], researchers used the apparatus of mathematical modeling to construct a mathematical model of catalyst activity in the process of catalytic dewaxing.

Of course, these works constitute a small part of the studies devoted to obtaining WDF. As a rule, each scientific work considers one of the aspects of obtaining WDF. In our study, we will consider two stages of obtaining WDF: dewaxing on zeolite and compounding (mixing the starting components without going through chemical reactions).

The main research methods: retrospective analysis of thr mathematical models of obtaining WDF, synthesis, comparison, the methods of linear programming.

\section{Results}

The main model assumptions and at the same time the scheme for constructing the MMWDF:

1. The first stage of obtaining WDF is dewaxing of a mixture of hydrotreated diesel fraction $200-320^{\circ} \mathrm{C}$ (hereinafter referred to as DF1, or $\mathrm{x} 1$ ), hydrogen-containing gas (hereinafter referred to as $\mathrm{HCG}$, or $\mathrm{x} 2$ ), hydrotreated WDF (hereinafter referred to as HTWDFd, or $\mathrm{x} 3$ ). The process is calculated for the target raw material, i.e. with the 
exception of HCG. In this case, HCG is considered to be the medium in which paraffin are released on zeolites. The yield of products depends only on the target raw material.

2. We compose the material balance equations for the first stage.

3. The second stage of obtaining WDF is compounding of denormalizate (hereinafter referred to as $\mathrm{x} 4$ ), diesel fraction $150-310{ }^{\circ} \mathrm{C}$ (hereinafter referred to as $\mathrm{DF} 2$, or $\mathrm{x} 8$ ), hydrotreated WDF (x9).

4. We draw up a system of restrictions on the quality of the received WDF (x10).

5. We write down the objective function (hereinafter referred to OF) and maximize profit. Let's schematically represent the first stage in Fig. 1.

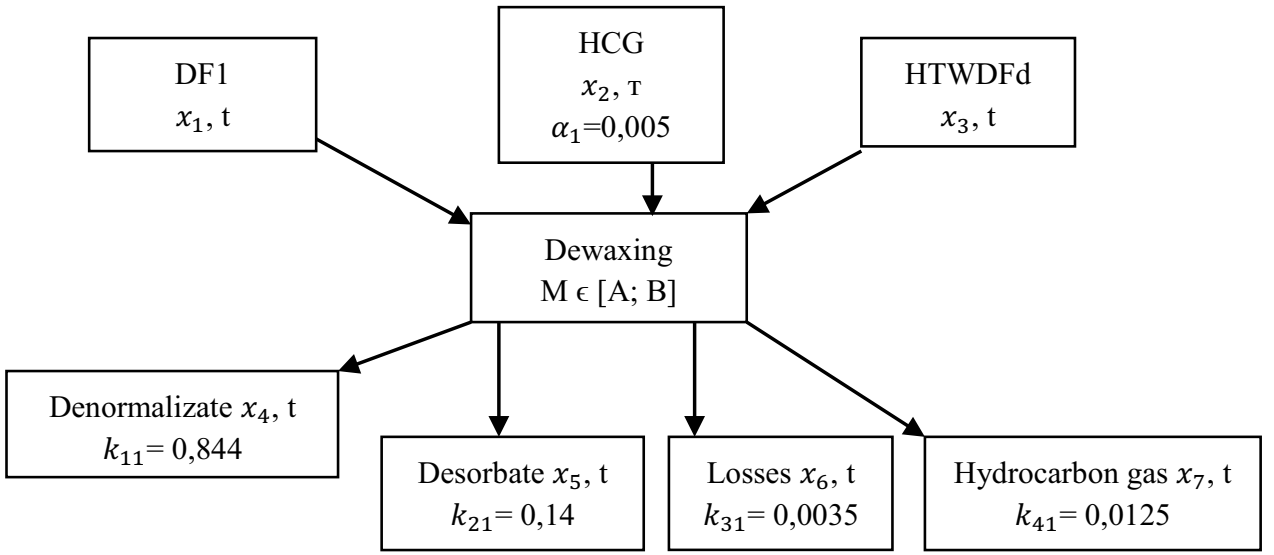

Fig. 1. Scheme of the first stage of obtaining WDF.

$$
\begin{gathered}
x_{1}+x_{2}+x_{3}+x_{4}+x_{5}+x_{6}+x_{7} \\
x_{2}=\alpha_{1}\left(x_{1}+x_{3}\right) \\
x_{4}-k_{1}\left(x_{1}+x_{3}\right)=0 \\
x_{5}-k_{2}\left(x_{1}+x_{3}\right)=0 \\
x_{6}-k_{3}\left(x_{1}+x_{3}\right)=0 \\
x_{7}-k_{4}\left(x_{1}+x_{3}\right)=0 \\
k_{1}+k_{2}+k_{3}+k_{4}-1=0 \\
x_{1}+x_{3} \leq A \\
x_{1}+x_{3} \leq B
\end{gathered}
$$

where:

$k_{1}, k_{2}, k_{3}, k_{4}$ are selection factors for the first stage;

A, B are boundaries of the capacity of the dewaxing unit;

$\mathrm{A}=1176$ tons, $\mathrm{B}=2256$ tons.

(2) is a mass balance equation;

(3) represents HCG as a share of the total mass of DF1 and HTWDFd

(4) is the equation of the output of denormalizate;

(5) is the equation of desorbate yield;

(6) is the equation of loss yield;

(7) is the equation of hydrocarbon gas yield;

(8) is the sum of the shares $(=1)$;

(9) and (10) are the limitations on the capacity of the dewaxing unit. 
For the first stage, there is no need to set the ratio of the components DF1 and HTWDFd, since they (within the framework of this process) have similar properties and can be mixed in any proportion.

Let us schematically represent the second stage in Fig. 2.

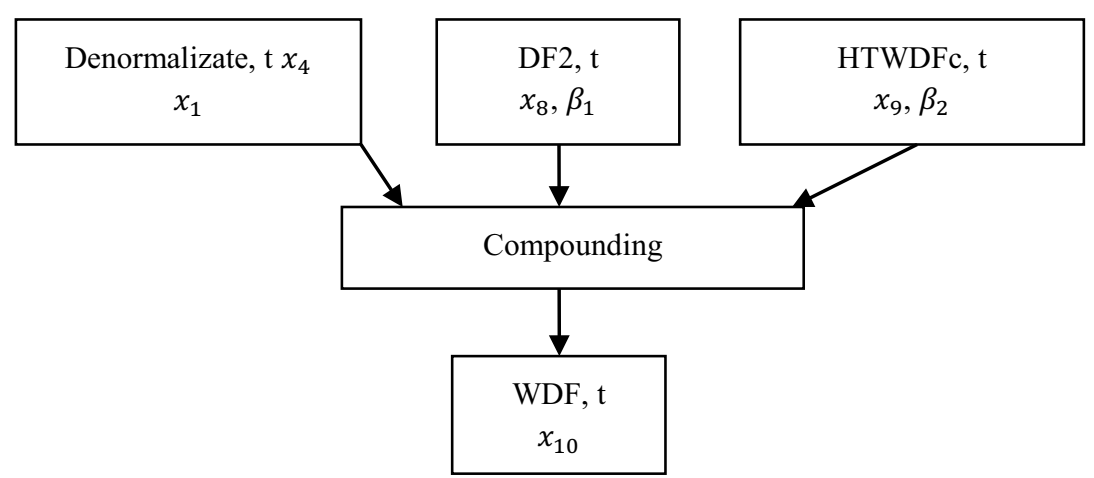

Fig. 2. Scheme of the second stage of obtaining WDF.

Denormalizate $\mathrm{x} 4$, obtained in the first stage, participates in the second stage compounding. Moreover, the number of DF2 (x8) and HTWDFc (x9) can be set as the shares of the amount of denormalizate $\mathrm{x} 4$.

Because of the second stage, it is required to reach the specified quality of the WDF. Quality indicators are represented in table 2.

Table 2. Quality indicators of components for compounding and WDF.

\begin{tabular}{|c|c|c|c|c|}
\hline No. & Substance & $\mathbf{\rho , ~} \mathbf{k g} / \mathbf{m 3}$ & $\mathbf{S}, \mathbf{p p m}$ & Flash point, ${ }^{\circ} \mathbf{C}$ \\
\hline 1 & Denormalizate $(\mathrm{x} 4)$ & $\rho_{4}=832.4$ & $s_{4}=2$ & $t_{4}=72$ \\
\hline 2 & HTWDFc (x9) & $\rho_{9}=786.4$ & $s_{9}=10$ & $t_{9}=34.5$ \\
\hline 3 & DF2 (x8) & $\rho_{8}=798$ & $s_{8}=1.3$ & $t_{8}=41$ \\
\hline 4 & WDF (x10) & $\begin{array}{c}\rho_{\min }=800 \\
\rho_{\max }=840\end{array}$ & $s_{10}=10$ & $t_{10}=40$ \\
\hline
\end{tabular}

$$
\begin{gathered}
x_{4}+x_{8}+x_{9}=x_{10} \\
x_{8}=\beta_{1} x_{4} \\
x_{9}=\beta_{2} x_{4} \\
s_{10} x_{10}-s_{4} x_{4}-s_{8} x_{8}-s_{9} x_{9} \leq 0 \\
x_{10} t_{10}=x_{4} t_{4}+x_{8} t_{8}+x_{9} t_{9} \\
x_{10} \rho_{\text {min }} \leq x_{4} \rho_{4}+x_{8} \rho_{8}+x_{9} \rho_{9} \\
x_{10} \rho_{\max } \leq x_{4} \rho_{4}+x_{8} \rho_{8}+x_{9} \rho_{9}
\end{gathered}
$$

where:

(11) is a weight balance;

(12) represents DF2 as a share of denormalizate;

(13) represents HTWDFc as a share of denormalizate;

(14) is a condition for sulfur content WDF;

(15) is a balance by conventional ton-degrees;

(16) is a condition for $\rho$ min in terms of conditional ton-density;

(17) is a condition for pmax in terms of conditional ton-density.

From $(12,13,14)$ : 


$$
x_{10}-x_{4}\left(1+\beta_{1}+\beta_{2}\right)
$$

Taking into account $(12,13)$, we rewrite $(14,15,16,17)$ in the following form:

$$
\begin{gathered}
s_{10} x_{10}-x_{4}\left(s_{4}+s_{8} \beta_{1}+s_{9} \beta_{2}\right) \leq 0 \\
x_{10} t_{10}-x_{4}\left(t_{4}+t_{8} \beta_{1}+t_{9} \beta_{2}\right)=0 \\
x_{10} \rho_{\text {min }}-x_{4}\left(\rho_{4}+\rho_{8} \beta_{1}+\rho_{9} \beta_{2}\right) \leq 0 \\
x_{10} \rho_{\text {max }}-x_{4}\left(\rho_{4}+\rho_{8} \beta_{1}+\rho_{9} \beta_{2}\right) \geq 0
\end{gathered}
$$

The objective function can be written as:

$$
O F=\rho_{10} x_{10}+\rho_{5} x_{5}+\rho_{7} x_{7}-c_{1} x_{1}-c_{3} x_{3}-c_{8} x_{8}-c_{9} x_{9} \rightarrow \max
$$

where:

$\rho_{10}, \rho_{5}, \rho_{7}$ are the prices of WDF, desorbate and hydrocarbon gas, rubles/ton;

$c_{1}, c_{3}, c_{3}, c_{9}$ are the costs of production and transportation per ton of the corresponding component, rubles/ton.

Accordingly, OF is aimed at maximizing the company's profits, taking into account production and logistics costs. Then the mathematical model of obtaining WDF is as follows:

$$
\begin{gathered}
\text { OF }=\rho_{10} x_{10}+\rho_{5} x_{5}+\rho_{7} x_{7}-c_{1} x_{1}-c_{3} x_{3}-c_{8} \beta_{1} x_{4}-c_{9} \beta_{2} x_{4} \rightarrow \max \\
x_{4}-k_{1}\left(x_{1}+x_{3}\right)=0 \\
x_{5}-k_{2}\left(x_{1}+x_{3}\right)=0 \\
x_{7}-k_{4}\left(x_{1}+x_{3}\right)=0 \\
x_{10}-x_{4}\left(1+\beta_{1}+\beta_{2}\right) \\
s_{10} x_{10}-x_{4}\left(s_{4}+s_{8} \beta_{1}+s_{9} \beta_{2}\right) \leq 0 \\
x_{10} t_{10}-x_{4}\left(t_{4}+t_{8} \beta_{1}+t_{9} \beta_{2}\right)=0 \\
x_{10} \rho_{\min }-x_{4}\left(\rho_{4}+\rho_{8} \beta_{1}+\rho_{9} \beta_{2}\right) \leq 0 \\
x_{10} \rho_{\max }-x_{4}\left(\rho_{4}+\rho_{8} \beta_{1}+\rho_{9} \beta_{2}\right) \geq 0 \\
x_{1}+x_{3} \leq A \\
x_{1}+x_{3} \leq B
\end{gathered}
$$

According to the authors, to solve the proposed MMWDF, it is possible to apply methods for solving linear optimization problems $[12,13]$.

\section{Discussion}

A mathematical model for the production of winter diesel fuel has been obtained. This mathematical model considers two stages of production: dewaxing and compounding.

Mathematical calculations describing the stage of dewaxing are based on linear dependencies. So it is proposed to consider that the components obtained after dewaxing on zeolite of a mixture of diesel fraction $200-320^{\circ}$, hydrogen-containing gas and hydrotreated winter diesel fuel are determined through the selection factor from the total mass of diesel fraction $200-320^{\circ}$ and hydrotreated winter diesel fuel intended for dewaxing. Hydrogencontaining gas acts as a working medium that promotes dewaxing on the zeolite. Accordingly, the main components at this stage are diesel fraction $200-320^{\circ}$ and 
hydrotreated winter diesel fuel intended for dewaxing. The amount of hydrogen-containing gas is set as a part $(\alpha 1)$ of the sum of the total mass of the diesel fraction $200-320^{\circ}$ and hydrotreated winter diesel fuel intended for dewaxing. The sum of the selection coefficients at the dewaxing stage exceeds 1 by exactly $\alpha 1$.

Mathematical calculations describing the compounding stage are also based on linear dependencies. Moreover, the main raw material for compounding is a normalizer. Secondary components for compounding (in the mathematical aspect) are diesel fraction $150-310^{\circ}$ and hydrotreated winter diesel fuel designed for compounding. They are calculated from the amount of denormalizate in proportion to the coefficients $\beta_{1}$ and $\beta_{2}$.

The mathematical model for obtaining winter diesel fuel also takes into account quality requirements. Linear dependencies also work here, which determine the conditions for the sulfur content in winter diesel fuel, conditions for the flash point, conditions for the density of winter diesel fuel.

Further, the mathematical model includes logistic indicators and, thereby, describes the production of winter diesel fuel, taking into account quality and logistics indicators [6-9]. The objective function is aimed at maximizing profits, which is important in a market economy.

According to the authors, this mathematical model almost completely covers the technological process of obtaining winter diesel fuel. Almost completely, since there is not described beginner stage of this process, petroleum distillation in the distillation column. As a result of this initial stage, we obtain straight-run diesel fraction and straight-run winter diesel fuel (here we do not focus our attention on the minor in this study products of oil distillation).

The sources we have considered in the literature review allow us to understand how this model can be supplemented and refined. The sources considered here were rather related to individual elements of the process of obtaining winter diesel fuel, but did not cover the whole process together. In this study, according to the authors, this is done, the whole process is covered. In an effort to capture the essence of the technological process for obtaining winter diesel fuel, the authors have sacrificed a number of parts. However, the available literature review allows us to supplement the existing mathematical model. At the same time, it should immediately be taken into account that the integration of many details into the existing model will force the sacrifice of linearity for the sake of thoroughness.

The papers [10], [11] focus on catalytic dewaxing. It is reasonable to assume that already at the stage of adding catalytic dewaxing processes to the existing mathematical model for obtaining winter diesel fuel, nonlinearity will appear. This nonlinearity will probably be to some extent.

The work [12] proposes to intensify the compounding process. You can also take this process into account in the existing mathematical model for obtaining winter diesel fuel. Probably, the equations of heat and mass transfer can be applied.

The work [13] indicates the possibility of adding cetane number restrictions to the existing mathematical model for obtaining winter diesel fuel.

The works [14], [15] indicate the possibility of adding a mathematical description of the catalytic desulfurization process to the existing mathematical model for obtaining winter diesel fuel. The nonlinearity of the original model will grow even more.

The work [16] suggests increasing the intensity of dewaxing by redistributing hydrogencontaining gas between the blocks of the unit. Using the outline of communicating capacities, a system of linear differential equations can be drawn up. The authors believe that in this case it will be the simplest description of the process.

As we can see, only these sources will significantly complicate the existing mathematical model for obtaining winter diesel fuel. Therefore, the benefits and costs of this complication will have to be further evaluated. 
So, the proposed mathematical model can be made closer to reality. For this, according to the authors, conditions should be added related at least to:

1. desulfurization of components;

2. catalytic desulfurization;

3. catalytic dewaxing.

The significance of the obtained mathematical model, according to the authors, lies in the fact that it can be easily verified in production conditions. It can be modified, supplemented and subsequently applied. You can use it to draw up a financial and economic model of the operation of an oil refinery and to integrate it into a complex of an automated control system for technological processes at an oil refinery. The authors believe that this mathematical model for the production of winter diesel fuel can serve as the core of such automated control system.

\section{Conclusions}

An approach to digitalization of the oil refining technological processes is proposed. According to the authors, to digitize them it is first necessary to build a mathematical model of the corresponding technological process. Further, this model will become the basis of the algorithm for the process control system.

The mathematical model for obtaining winter diesel fuel (WDF) has been built. This model considers two stages of WDF production: dewaxing and compounding. The objective function maximizes the profit of an enterprise, taking into account production and logistics costs.

A method for solving the constructed mathematical model is proposed, a kind of the simplex method costs.

\section{References}

1. Russian Statistical Yearbook: Stat.book (Rosstat, 2018)

2. N.S. Belinskaya, E.V. Frantsina, Models, systems, networks in economics, engineering, nature and society 2(6), 145-148 (2013)

3. N.S. Belinskay, Current Organic Synthesis 14(3), 365-371 (2017)

4. E.D. Ivanchina, E.N. Ivashkina, V.A. Chuzlov, Chemical Engineering Journal 383, 121283 (2020)

5. M.V. Kirgina, I. Bogdanov, N.S. Belinskaya, Oil \& Gas Science and Technology Revue d'IFP Energies Nouvelles 75, 31-35 (2020)

6. V. Vilken, O. Kalinina, S. Barykin, E. Zotova, IOP Conference Series: Materials Science and Engineering (2019) https://doi.org/10.1088/1757-899X/497/1/012037

7. D. Egorov, A. Levina, S. Kalyazina, P. Schuur, B. Gerrits, Lecture Notes in Networks and Systems 157, 201-209 (2021)

8. I.V. Ilin, A.V. Izotov, S.V. Shirokova, O.V. Rostova, A.I. Levina, Proceedings of 20th IEEE International Conference on Soft Computing and Measurements, SCM 2017 7970732, 812-814 (2017) DOI: 10.1109/SCM.2017.7970732

9. A. Anisiforov, A. Dubgorn, A. Lepekhin, E3S Web of Conferences 110, 02051 (2019)

10. N.S. Belinskaya, E.V. Frantsina, E.D. Ivanchina, Chemical Engineering Journal (2017) https://doi.org/10.1016/j.cej.2017.04.033

11. N.S. Belinskaya, E.V. Frantsina, E.D. Ivanchina, Catalysis Today 329, 214-220 (2019) 
12. E.D. Ivanchina, I.M. Dolganov, V.A. Chuzlov, N.S. Belinskaya, Chemical Engineering \& Processing: Process Intensification 122, 415-424 (2017)

13. M.V. Kirgina, I. Bogdanov, A.A. Altynov, N.S. Belinskaya, Petroleum and Coal 61(1), 110-119 (2019)

14. E.V. Frantsina, Petroleum and Coal 61(1), 74-80 (2019)

15. N.I. Krivtsova, Petroleum Science and Technology 37(2), 181-189 (2019)

16. N.S. Belinskaya, Petroleum and Coal 60(1), 7-13 (2018)

17. E.V. Frantsina, N.S. Belinskaya, E.D. Ivanchina, Korean Journal of Chemical Engineering 35(2), 337-347 (2018) https://doi.org/10.1007/s11814-017-0284-X

18. E.V. Frantsina, Petroleum Science and Technology (2018) https://doi.org 10.1080/10916466.2018.1504068

19. E.V. Frantsina, N.S. Belinskaya, D.A. Afanaseva, Petroleum and Coal 59(1), 82-88 (2017)

20. E.V. Frantsina, N.S. Belinskaya, E.D. Ivanchina, A.S. Lutsenko, Petroleum and Coal 59(5), 729-735 (2017)

21. A. Rudskoy, I. Ilin, Proceedings of the 33rd International Business Information Management Association Conference, IBIMA 2019: Education Excellence and Innovation Management through Vision 2020, 8643-8649 (2019) 